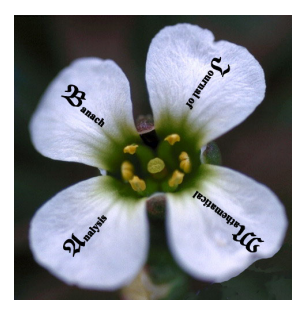

Banach J. Math. Anal. 2 (2008), no. 2, 85-93

B anach J ournal of $\mathbf{M}_{\text {athematical }} \mathbf{A}_{\text {nalysis }}$

ISSN: $1735-8787$ (electronic)

http://www.math-analysis.org

\title{
THREE-PARAMETER WEIGHTED HARDY TYPE INEQUALITIES
}

\author{
R. OINAROV ${ }^{1 *}$ AND A. KALYBAY ${ }^{2}$ \\ This paper is dedicated to Professor Josip E. Pečarić
}

Submitted by S.-M. Jung

Abstract. For $0<r<\infty$ and $1 \leq p \leq q<\infty$ we find necessary and sufficient conditions for the validity of the following inequality:

$$
\left(\int_{a}^{b} u(x)\left(\int_{a}^{x}|g(x)-g(t)|^{r} w(t) d t\right)^{\frac{q}{r}} d x\right)^{\frac{1}{q}} \leq C\left(\int_{a}^{b} v(x)\left|g^{\prime}(x)\right|^{p} d x\right)^{\frac{1}{p}},
$$

where $u(\cdot), v(\cdot)$, and $w(\cdot)$ are weight functions.

\section{INTRODUCTION AND PRELIMINARIES}

Let $0<r, p, q<\infty$ and $-\infty \leq a<b \leq+\infty$. Suppose that $u(\cdot), v(\cdot)$, and $w(\cdot)$ are weight functions, i.e., positive and locally integrable on $(a, b)$.

We consider the following inequalities:

$$
\left(\int_{a}^{b} u(x)\left(\int_{a}^{x}|g(x)-g(t)|^{r} w(t) d t\right)^{\frac{q}{r}} d x\right)^{\frac{1}{q}} \leq C\left(\int_{a}^{b} v(x)\left|g^{\prime}(x)\right|^{p} d x\right)^{\frac{1}{p}}
$$

Date: Received: 14 April 2008; Accepted: 18 June 2008.

* This research work was supported by the INTAS grant Ref. Nr 05-1000008-8157.

2000 Mathematics Subject Classification. Primary 26D10, 26D15; Secondary 46E15, 46E30.

Key words and phrases. Inequalities, Hardy type inequalities, weight functions. 


$$
\left(\int_{a}^{b} u(x)\left(\int_{x}^{b}|g(t)-g(x)|^{r} w(t) d t\right)^{\frac{q}{r}} d x\right)^{\frac{1}{q}} \leq C\left(\int_{a}^{b} v(x)\left|g^{\prime}(x)\right|^{p} d x\right)^{\frac{1}{p}} .
$$

If we put that $g^{\prime}(x)=f(x)$ for $x \in(a, b)$, then the inequalities (1.1) and (1.2) have the following forms:

$$
\begin{aligned}
& \left(\int_{a}^{b} u(x)\left(\int_{a}^{x}\left|\int_{t}^{x} f(s) d s\right|^{r} w(t) d t\right)^{\frac{q}{r}} d x\right)^{\frac{1}{q}} \leq C\left(\int_{a}^{b} v(x)|f(x)|^{p} d x\right)^{\frac{1}{p}}, \\
& \left(\int_{a}^{b} u(x)\left(\int_{x}^{b}\left|\int_{x}^{t} f(s) d s\right|^{r} w(t) d t\right)^{\frac{q}{r}} d x\right)^{\frac{1}{q}} \leq C\left(\int_{a}^{b} v(x)|f(x)|^{p} d x\right)^{\frac{1}{p}},
\end{aligned}
$$

respectively.

In the inequalities (1.3) and (1.4) without loss of generality we suppose that $f \geq 0$.

In this paper we study the inequalities $\sqrt{1.1})(\sqrt{1.3}))$ and $\sqrt{1.2})((1.4))$ in the case $0<r<\infty$ and $1 \leq p \leq q<\infty$.

Let $(\alpha, \beta) \subset(a, b)$ and $\frac{1}{p}+\frac{1}{p^{\prime}}=1$. Assume

$$
\begin{gathered}
\underline{v}(\alpha, \beta)=\operatorname{ess} \inf _{\alpha<t<\beta} v(t), U(\alpha, \beta)=\left(\int_{\alpha}^{\beta} u(x) d x\right)^{\frac{1}{q}}, \\
A^{+}(\alpha, \beta)=\sup _{x \in(\alpha, \beta)}\left(\int_{x}^{\beta} w(t) d t\right)^{\frac{1}{r}}\left(\int_{\alpha}^{x} v^{1-p^{\prime}}(s) d s\right)^{\frac{1}{p^{\prime}}}, \\
B^{+}(\alpha, \beta)=\left(\int_{\alpha}^{\beta}\left(\int_{x}^{\beta} w(t) d t\right)^{\frac{r}{p-r}}\left(\int_{\alpha}^{x} v^{1-p^{\prime}}(s) d s\right)^{\frac{r(p-1)}{p-r}} w(x) d x\right)^{\frac{p-r}{p r}}, \\
D^{+}(\alpha, \beta)=\left(\int_{\alpha}^{\beta}\left(\int_{x}^{\beta} w(t) d t\right)^{\frac{r}{1-r}}(\underline{v}(\alpha, x))^{\frac{r}{r-1}} w(x) d x\right)^{\frac{1-r}{r}}, \\
A^{-}(\alpha, \beta)=\sup _{x \in(\alpha, \beta)}\left(\int_{\alpha}^{x} w(t) d t\right)^{\frac{1}{r}}\left(\int_{x}^{\beta} v^{1-p^{\prime}}(s) d s\right)^{\frac{1}{p^{\prime}}},
\end{gathered}
$$




$$
\begin{gathered}
B^{-}(\alpha, \beta)=\left(\int_{\alpha}^{\beta}\left(\int_{\alpha}^{x} w(t) d t\right)^{\frac{r}{p-r}}\left(\int_{x}^{\beta} v^{1-p^{\prime}}(s) d s\right)^{\frac{r(p-1)}{p-r}} w(x) d x\right)^{\frac{p-r}{p r}}, \\
D^{-}(\alpha, \beta)=\left(\int_{\alpha}^{\beta}\left(\int_{\alpha}^{x} w(t) d t\right)^{\frac{r}{1-r}}(\underline{v}(x, \beta))^{\frac{r}{r-1}} w(x) d x\right)^{\frac{1-r}{r}}, \\
J^{+}(\alpha, \beta)=\sup _{f \geq 0} \frac{\left(\int_{\alpha}^{\beta} w(t)\left(\int_{\alpha}^{t} f(s) d s\right)^{r} d t\right)^{\frac{1}{r}}}{\left(\int_{\alpha}^{\beta} v(x) f^{p}(x) d x\right)^{\frac{1}{p}}} \\
J^{-}(\alpha, \beta)=\sup _{f \geq 0} \frac{\left(\int_{\alpha}^{\beta} w(t)\left(\int_{t}^{\beta} f(s) d s\right)^{r} d t\right)^{\frac{1}{r}}}{\left(\int_{\alpha}^{\beta} v(x) f^{p}(x) d x\right)^{\frac{1}{p}}}
\end{gathered}
$$

By now owing to a series of works there are known the exact in order estimates of the values $J^{+}$and $J^{-}$for the different relations between the parameters $0<$ $r<\infty$ and $p \geq 1$. Review of these works, corresponding references and obtained results are given in the monograph [1]. From [1] and some more exact results from [2] we have

Lemma A. (i) If $1 \leq p \leq r<\infty$, then

$$
A^{ \pm}(\alpha, \beta) \leq J^{ \pm}(\alpha, \beta) \leq p^{\frac{1}{r}}\left(p^{\prime}\right)^{\frac{1}{p^{\prime}}} A^{ \pm}(\alpha, \beta) .
$$

(ii) If $0<r<p$ and $1<p<\infty$, then

$$
\left(p^{\prime}\right)^{\frac{1}{p^{\prime}}} r^{\frac{1}{p}}\left(1-\frac{r}{p}\right) B^{ \pm}(\alpha, \beta) \leq J^{ \pm}(\alpha, \beta) \leq\left(\frac{p}{p-r}\right)^{\frac{p-r}{p r}} p^{\frac{1}{p}}\left(p^{\prime}\right)^{\frac{1}{p^{\prime}}} B^{ \pm}(\alpha, \beta) .
$$

(iii) If $0<r<1=p$, then

$$
r(1-r) D^{ \pm}(\alpha, \beta) \leq J^{ \pm}(\alpha, \beta) \leq(1-r)^{\frac{1-r}{r}} D^{ \pm}(\alpha, \beta) .
$$

Let us notice that in [1] and [2] for the case $0<r<1$ the estimates (1.6) and (1.7) are given only for $J^{+}$, however, by changing of integration variable they can be easily reduced to $J^{-}$(see [1], page 53).

In the sequel the symbol $A \ll B$ means $A \leq C B$ with some constant $C>0$ that depends only on the parameters $p, r$, and $q$. The notation $A \approx B$ asserts the existence of the two - sided estimate $A \ll B \ll A$. Moreover, we suppose that the expressions of the form $\frac{0}{0}, \frac{\infty}{\infty}$, and $0 \cdot \infty$ are taken equal to zero. 


\section{MAin RESUlts}

Theorem 2.1. The inequality (1.1) (1.3)) holds if and only if

(i) $E=\sup _{z>0} U(z, b) A^{-}(a, z)<\infty$ for $1 \leq p \leq \min \{r, q\}<\infty$;

(ii) $E=\sup _{z>0} U(z, b) B^{-}(a, z)<\infty$ for $0<r<p$ and $1<p \leq q<\infty$;

(iii) $E=\sup _{z>0} U(z, b) D^{-}(a, z)<\infty$ for $0<r<1=p \leq q<\infty$ and $\underline{v}(\alpha, \beta)>0$ for any $\alpha, \beta: a<\alpha<\beta<b$.

Moreover, $E \approx C$, where $C$ is the best constant in 1.1) (1.3)).

Theorem 2.2. The inequality (1.2) (1.4)) holds if and only if

(i) $F=\sup _{z>0} U(a, z) A^{+}(z, b)<\infty$ for $1 \leq p \leq \min \{r, q\}<\infty$;

(ii) $F=\sup _{z>0} U(a, z) B^{+}(z, b)<\infty$ for $1<r<p$ and $1<p \leq q<\infty$;

(iii) $F=\sup _{z>0} U(a, z) D^{+}(z, b)<\infty$ for $0<r<1=p \leq q<\infty$ and $\underline{v}(\alpha, \beta)>0$ for any $\alpha, \beta: a<\alpha<\beta<b$.

Moreover, $F \approx C$, where $C$ is the best constant in (1.2) (1.4)).

We shall prove only Theorem 2.1 because Theorem 2.2 can be proved similarly.

Proof of Theorem 2.1. Since the inequalities (1.1) and (1.3) are equivalent and moreover, their best constants coincide we shall prove Theorem 2.1 only for (1.3).

Necessity. Suppose that (1.3) is valid. Let $z \in(a, b)$ and $f:(a, z) \rightarrow R$ be an arbitrary function, for which $\int_{a}^{z} v(t)|f(t)|^{p} d t<\infty$. Suppose that

$$
f_{z}(t)=\left\{\begin{array}{l}
f(t), a<t<z \\
0, z \leq t<b
\end{array}\right.
$$

By substitution of the function $f_{z}$ in $(1.3)$ we have that

$$
\left(\int_{z}^{b} u(x) d x\right)^{\frac{1}{q}}\left(\int_{a}^{z}\left|\int_{t}^{z} f(s) d s\right|^{r} w(t) d t\right)^{\frac{1}{r}} \leq C\left(\int_{a}^{z} v(x)|f(x)|^{p} d x\right)^{\frac{1}{p}} .
$$

This yields that

$$
U(z, b) J^{-}(a, z) \leq C, \forall z \in(a, b) .
$$

From (2.1) and Lemma $\underline{\mathrm{A}}$ for all $z \in(a, b)$ we obtain that

$$
\begin{gathered}
U(z, b) A^{-}(a, z) \leq C \text { for } 1 \leq p \leq r<\infty \\
U(z, b) B^{-}(a, z) \ll C \text { for } 0<r<p \text { and } 1<p<\infty, \\
U(z, b) D^{-}(a, z) \ll C \text { for } 0<r<1=p .
\end{gathered}
$$

Therefore,

$$
E \ll C
$$


Sufficiency. Let $E<\infty$ and $f \geq 0$. For any integer $k$ we define:

$$
x_{k}=\sup \left\{x \in(a, b): \int_{a}^{x}\left(\int_{t}^{x} f(s) d s\right)^{r} w(t) d t \leq 2^{r k}\right\} .
$$

It is obvious that $x_{k} \leq x_{k+1}$, however, if $x_{k}<b$, then $x_{k}<x_{k+1} \leq b$. Hence,

$$
\int_{a}^{x_{k}}\left(\int_{t}^{x_{k}} f(s) d s\right)^{r} w(t) d t=2^{r k} \text { and } \int_{a}^{x_{k+1}}\left(\int_{t}^{x_{k+1}} f(s) d s\right)^{r} w(t) d t \leq 2^{r(k+1)} \text {. }
$$

Let $I_{k}=\left[x_{k}, x_{k+1}\right)$. Then

$$
(a, b)=\bigcup_{k}\left[x_{k}, x_{k+1}\right) .
$$

Let $x_{k}<b$. If $r \geq 1$, then applying Minkowski's inequality, we find that

$$
\begin{gathered}
2^{k-1}=2^{k}-2^{k-1}=\left(\int_{a}^{x_{k}}\left(\int_{t}^{x_{k}} f(s) d s\right)^{r} w(t) d t\right)^{\frac{1}{r}} \\
\left.-\left(\int_{t}^{x_{k-1}}\left(\int_{t}^{x_{k-1}} f(s) d s\right)^{\frac{1}{r}} w(t) d t\right)_{c_{k-1}}^{x_{k}}\left(\int_{t}^{x_{k}} f(s) d s\right)^{r} w(t) d t\right)^{\frac{1}{r}} \\
+\left(\int_{a}^{x_{k-1}}\left(\int_{t}^{x_{k-1}} f(s) d s+\int_{x_{k-1}}^{x_{k}} f(s) d s\right)^{r} w(t) d t\right)^{\frac{1}{r}} \\
\leq\left(\int_{x_{k-1}}^{x_{k}}\left(\int_{t}^{x_{k-1}} f(s) d s\right)^{x_{k}}\left(\int_{t}^{x_{k-1}} f(s) d s\right)^{r} w(t) d t\right)^{\frac{1}{r}}+\left(\int_{a}^{\frac{1}{r}} w(t) d t\right)^{\frac{1}{r}} \int_{x_{k-1}}^{x_{k}} f(s) d s .
\end{gathered}
$$

In the case $0<r<1$ we get that

$$
\begin{gathered}
2^{r(k-1)}=\frac{1}{2^{r}-1}\left(2^{r k}-2^{r(k-1)}\right)=\frac{1}{2^{r}-1}\left(\int_{x_{k-1}}^{x_{k}}\left(\int_{t}^{x_{k}} f(s) d s\right)^{r} w(t) d t\right. \\
\left.+\int_{a}^{x_{k-1}}\left(\int_{t}^{x_{k-1}} f(s) d s+\int_{x_{k-1}}^{x_{k}} f(s) d s\right)^{r} w(t) d t-\int_{a}^{x_{k-1}}\left(\int_{t}^{x_{k-1}} f(s) d s\right)^{r} w(t) d t\right)
\end{gathered}
$$




$$
\leq \frac{1}{2^{r}-1}\left(\int_{x_{k-1}}^{x_{k}}\left(\int_{t}^{x_{k}} f(s) d s\right)^{r} w(t) d t+\int_{a}^{x_{k-1}}\left(\int_{x_{k-1}}^{x_{k}} f(s) d s\right)^{r} w(t) d t\right) .
$$

From 2.3 and 2.4 we have that

$$
\begin{aligned}
& T \equiv \int_{a}^{b} u(x)\left(\int_{a}^{x}\left(\int_{t}^{x} f(s) d s\right)^{r} w(t) d t\right)^{\frac{q}{r}} d x \\
\leq & \sum_{k} \int_{x_{k}}^{x_{k+1}} u(x) d x\left(\int_{a}^{x_{k+1}}\left(\int_{t}^{x_{k+1}} f(s) d s\right)^{r} w(t) d t\right)^{\frac{q}{r}} \\
\leq & \sum_{k} 2^{q(k+1)} \int_{x_{k}}^{x_{k+1}} u(x) d x \leq 2^{2 q} \sum_{k} 2^{q(k-1)} \int_{x_{k}}^{x_{k+1}} u(x) d x .
\end{aligned}
$$

Here and in the sequel the summation over $k$ is with respect to the set $\{k$ : $\left.I_{k} \neq \emptyset\right\}$.

Let $r \geq 1$. Then from (2.5) and (2.7) it follows that

$$
\begin{gathered}
T \leq 2^{2 q} \sum_{k}\left(\left(\int_{x_{k-1}}^{x_{k}}\left(\int_{t}^{x_{k}} f(s) d s\right)^{r} w(t) d t\right)^{\frac{1}{r}}\right. \\
\left.+\left(\int_{a}^{x_{k-1}} w(t) d t\right)_{x_{k-1}^{\frac{1}{r}}}^{x_{k}} f(s) d s\right)^{q} \int_{x_{k}}^{x_{k+1}} u(x) d x \\
\ll \sum_{k}\left(\int_{x_{k-1}}^{x_{k}}\left(\int_{t}^{x_{k}} f(s) d s\right)^{r} w(t) d t\right)^{\frac{q}{r}} u(x) d x \\
+\sum_{k}\left(\int_{a}^{x_{k+1}} w(t) d t\right)_{x_{k}}^{\frac{q}{r}}\left(\int_{x_{k-1}}^{x_{k}} f(s) d s\right)_{x_{x_{k}}}^{q} u(x) d x=T_{1}+T_{2} .
\end{gathered}
$$

In the case $0<r<1$ due to $(2.6)$ and $(2.7)$ we have that

$$
T \ll \sum_{k}\left(2^{r(k-1)}\right)^{\frac{q}{r}} \int_{x_{k}}^{x_{k+1}} u(x) d x \leq\left(\frac{1}{2^{r}-1}\right)^{\frac{q}{r}} \sum_{k}\left(\int_{x_{k-1}}^{x_{k}}\left(\int_{t}^{x_{k}} f(s) d s\right)^{r} w(t) d t\right.
$$




$$
\begin{gathered}
\left.+\int_{a}^{x_{k-1}} w(t) d t\left(\int_{x_{k-1}}^{x_{k}} f(s) d s\right)^{r}\right)^{\frac{q}{r}} \int_{x_{k}}^{x_{k+1}} u(x) d x \\
\ll \sum_{k}\left(\int_{x_{k-1}}^{x_{k}}\left(\int_{t}^{x_{k}} f(s) d s\right)^{r} w(t) d t\right)^{\frac{q}{r}} \int_{x_{k}}^{x_{k+1}} u(x) d x \\
+\sum_{k}\left(\int_{a}^{x_{k-1}} w(t) d t\right)^{\frac{q}{r}}\left(\int_{x_{k-1}}^{x_{k}} f(s) d s\right)^{q} \int_{x_{k}}^{x_{k+1}} u(x) d x=T_{1}+T_{2} .
\end{gathered}
$$

Now, we shall estimate $T_{1}$ and $T_{2}$ for each of the cases (i), (ii), and (iii) separately.

Let $1 \leq p \leq \min \{r, q\}<\infty$. Using the upper estimate of 1.5 we obtain that

$$
\begin{gathered}
T_{1} \ll \sum_{k}\left(A^{-}\left(x_{k-1}, x_{k}\right) U\left(x_{k}, x_{k+1}\right)\right)^{q}\left(\int_{x_{k-1}}^{x_{k}} v(t)|f(t)|^{p} d t\right)^{\frac{q}{p}} \\
\leq \sum_{k}\left(A^{-}\left(a, x_{k}\right) U\left(x_{k}, b\right)\right)^{q}\left(\int_{x_{k-1}}^{x_{k}} v(t)|f(t)|^{p} d t\right)^{\frac{q}{p}} \leq E^{q} \sum_{k}\left(\int_{x_{k-1}}^{x_{k}} v(t)|f(t)|^{p} d t\right)^{\frac{q}{p}} \\
\leq E^{q}\left(\sum_{k} \int_{x_{k-1}}^{x_{k}} v(t)|f(t)|^{p} d t\right)^{\frac{q}{p}} \leq E^{q}\left(\int_{a}^{b} v(t)|f(t)|^{p} d t\right)^{\frac{q}{p}} \cdot
\end{gathered}
$$

To estimate $T_{2}$ we use Hölder's inequality:

$$
\begin{aligned}
& T_{2} \leq \sum_{k}\left(\int_{a}^{x_{k-1}} w(t) d t\right)^{\frac{q}{r}}\left(\int_{x_{k-1}}^{x_{k}} v^{1-p^{\prime}}(t) d t\right)^{\frac{q}{p^{\prime}}} \int_{x_{k}}^{x_{k+1}} u(x) d x\left(\int_{x_{k-1}}^{x_{k}} v(t)|f(t)|^{p} d t\right)^{\frac{q}{p}}(2.11) \\
& \leq \sum_{k}\left(A^{-}\left(a, x_{k}\right) U\left(x_{k}, b\right)\right)^{q}\left(\int_{x_{k-1}}^{x_{k}} v(t)|f(t)|^{p} d t\right)^{\frac{q}{p}} \leq E^{q}\left(\int_{a}^{b} v(t)|f(t)|^{p} d t\right)^{\frac{q}{p}}(2.12)
\end{aligned}
$$

From 2.8$), 2.10$, and 2.12 it follows that in the case (i) the inequality (1.3) is correct with the estimate

$$
C \ll E,
$$

where $C$ is the best constant in $(1.3)$. 
Let $0<r<p$ and $1<p \leq q<\infty$. By using the upper estimate of $(1.6)$ we get that

$$
\begin{gathered}
T_{1} \ll \sum_{k}\left(B^{-}\left(x_{k-1}, x_{k}\right) U\left(x_{k}, x_{k+1}\right)\right)^{q}\left(\int_{x_{k-1}}^{x_{k}} v(t)|f(t)|^{p} d t\right)^{\frac{q}{p}} \\
\leq \sum_{k}\left(B^{-}\left(a, x_{k}\right) U\left(x_{k}, b\right)\right)^{q}\left(\int_{x_{k-1}}^{x_{k}} v(t)|f(t)|^{p} d t\right)^{\frac{q}{p}} \leq E^{q}\left(\int_{a}^{b} v(t)|f(t)|^{p} d t\right)^{\frac{q}{p}}(2 .
\end{gathered}
$$

To estimate $T_{2}$ we previously carry out the following calculations:

$$
\begin{gathered}
\left(\int_{a}^{x_{k-1}} w(t) d t\right)^{\frac{1}{r}}\left(\int_{x_{k-1}}^{x_{k}} v^{1-p^{\prime}}(t) d t\right)^{\frac{1}{p^{\prime}}} \\
\leq\left(\frac{p-r}{p} \int_{a}^{x_{k-1}}\left(\int_{a}^{x} w(t) d t\right)^{\frac{r}{p-r}} w(t) d t\right)^{\frac{p-r}{p r}}\left(\int_{x_{k-1}}^{x_{k}} v^{1-p^{\prime}}(t) d t\right)^{\frac{1}{p^{\prime}}} \\
\ll\left(\int_{a}^{x_{k-1}}\left(\int_{a}^{x} w(t) d t\right)^{\frac{r}{p-r}} w(t) d t\right)^{\frac{p-r}{p r}}\left(\int_{x_{k-1}}^{x_{k}} v^{1-p^{\prime}}(t) d t\right)^{\frac{1}{p^{\prime}}} \leq B^{-}\left(a, x_{k}\right) .
\end{gathered}
$$

From (2.11) and $(2.15)$ it follows that

$$
T_{2} \ll \sum_{k}\left(B^{-}\left(a, x_{k}\right) U\left(x_{k}, b\right)\right)^{q}\left(\int_{x_{k-1}}^{x_{k}} v(t)|f(t)|^{p} d t\right)^{\frac{q}{p}} \leq E^{q}\left(\int_{a}^{b} v(t)|f(t)|^{p} d t\right)^{\frac{q}{p}}(2 .
$$

From (2.8), 2.9), (2.14), and (2.16) we have that in the case (ii) the inequality (1.3) is correct with the estimate (2.13), where $C$ is the best constant in (1.3).

Let us turn to the case (iii). From the right - hand estimate of (1.7) we obtain that

$$
\begin{aligned}
T_{1} & \ll \sum_{k}\left(D^{-}\left(x_{k-1}, x_{k}\right) U\left(x_{k}, x_{k+1}\right)\right)^{q}\left(\int_{x_{k-1}}^{x_{k}} v(s) f(s) d s\right)^{q} \\
& \leq E^{q}\left(\sum_{k} \int_{x_{k-1}}^{x_{k}} v(s) f(s) d s\right)^{q} \leq E^{q}\left(\int_{a}^{b} v(s) f(s) d s\right)^{q} .
\end{aligned}
$$


In this case by the condition $\underline{v}(\alpha, \beta)>0$ for any $\alpha, \beta: a<\alpha<\beta<b$, therefore:

$$
\begin{aligned}
& T_{2}=\sum_{k} U^{q}\left(x_{k}, x_{k+1}\right)\left(\int_{a}^{x_{k-1}} w(t) d t\right)^{\frac{q}{r}} \frac{1}{\underline{v}^{q}\left(x_{k-1}, x_{k}\right)} \underline{v}^{q}\left(x_{k-1}, x_{k}\right)\left(\int_{x_{k-1}}^{x_{k}} f(s) d s\right)^{q} \\
& \ll \sum_{k} U^{q}\left(x_{k}, b\right)\left(\int_{a}^{x_{k-1}}\left(\int_{a}^{x} w(t) d t\right)^{\frac{r}{1-r}} w(t) d t\right)^{\frac{(1-r) q}{r}} \frac{1}{\underline{v}^{q}\left(x_{k-1}, x_{k}\right)}\left(\int_{x_{k-1}}^{x_{k}} v(s) f(s) d s\right)^{q} \\
& \leq \sum_{k}\left(U\left(x_{k}, b\right)\left(\int_{a}^{x_{k-1}}\left(\int_{a}^{x} w(t) d t\right)^{\frac{r}{1-r}} \underline{v}^{\frac{r}{r-1}}\left(t, x_{k}\right) w(t) d t\right)^{q}\left(\int_{x_{k-1}}^{x_{k}} v(s) f(s) d s\right)^{q}\right. \\
& \leq \sum_{k}\left(U\left(x_{k}, b\right) D^{-}\left(a, x_{k}\right)\right)^{q}\left(\int_{x_{k-1}}^{x_{k}} v(s) f(s) d s\right)^{q} \leq E^{q}\left(\int_{a}^{b} v(s) f(s) d s\right)^{q} .(2.18)
\end{aligned}
$$

From (2.9), 2.17), and (2.18) we have that the estimate 1.3$)$ is again correct with the estimate (2.13), where $C$ is the best constant.

Thus, in all three cases the estimate $(1.3)$ holds with the estimate 2.13 for the best constant $C$. This together with $(2.2)$ gives that $C \approx E$. The proof of Theorem 2.1 is complete.

\section{REFERENCES}

1. A. Kufner, L. Maligranda and L.-E. Persson, The Hardy inequality. About its history and some related results, Pilsen, 2007.

2. G. Sinnamon and V.D. Stepanov, The weighted Hardy inequality: new proofs and the case $p=1$, J. London Math. Soc. 54 (1996), 89-101.

1 Eurasian National University, Munaytpasov st. 5, Astana 010008, KazaKHSTAN.

E-mail address: o_ryskul@mail.ru

2 Kazakhstan Institute of Management, Economics and Strategic Research, Abay ave. 4, Almaty 050010, Kazakhstan.

E-mail address: aigerim_k@academset.kz 